\title{
Effect of retro walking versus balance training on pain and disability in patients with osteoarthritis of the knee: a randomized controlled trial
}

\author{
Vijaya Krishnan *i] and Krishna Pithadia
}

\begin{abstract}
Background: Osteoarthritis $(\mathrm{OA})$ is a chronic, degenerative, inflammatory musculoskeletal dysfunction. It is associated with pain, reduced functional capacity, and deficient quality of life posing a challenge for rehabilitation. The study objective was to determine the treatment protocol for effectively managing pain and functional disability of patients with OA knee.

Methods: This is a single-blinded study conducted in the outpatient department of a tertiary care hospital. This three-arm study consisted of 20 patients randomly allocated to each group, prearranged to receive conventional treatment (group A), conventional treatment with retro walking training (group B), and conventional treatment with balance training (group C) for a time frame of 6 weeks. Patients were screened prior to the pre-set eligibility criteria before inclusion in the study. Pain and functional disability were assessed the baseline and post-intervention.

Results: The findings reported $66.66 \%$ female participation. The results established statistical significance for both pain and functional disability across all the three groups for both intergroup and intragroup analysis. Group B demonstrated the most promising results.

Conclusion: The 6-week rehabilitation protocol comprising retro walking as an adjunct to conventional therapy resulted in greater pain reduction and enhanced functional performance of patients with OA knee. This benefits the community at large. All stakeholders should be encouraged to include retro walking in their routine life.

Trial registration: The University Hospital Medical Information Network Centre (UMIN-CTR), UMIN000044483. Retrospectively registered on June 09, 2021.
\end{abstract}

Keywords: Osteoarthritis, Knee, Balance, Retro walking, Rehabilitation, Public health

\section{Background}

The Osteoarthritis Research Society International (OARSI) has defined "osteoarthritis (OA) as a disorder involving movable joints characterized by cell stress and extracellular matrix degradation initiated by micro- and macro-injury that activates maladaptive repair responses including proinflammatory pathways of innate immunity; the disease

\footnotetext{
* Correspondence: victoryv2@yahoo.co.in

Department Musculoskeletal Physiotherapy, MGM College of Physiotherapy, Navi Mumbai, India
}

\section{Springer Open}

(c) The Author(s). 2021 Open Access This article is licensed under a Creative Commons Attribution 4.0 International License, which permits use, sharing, adaptation, distribution and reproduction in any medium or format, as long as you give appropriate credit to the original author(s) and the source, provide a link to the Creative Commons licence, and indicate if changes were made. The images or other third party material in this article are included in the article's Creative Commons licence, unless indicated otherwise in a credit line to the material. If material is not included in the article's Creative Commons licence and your intended use is not permitted by statutory regulation or exceeds the permitted use, you will need to obtain permission directly from the copyright holder. To view a copy of this licence, visit http://creativecommons.org/licenses/by/4.0/. joint tissue metabolism) followed by anatomic and/or physiologic derangements (characterized by cartilage degradation, bone remodeling, osteophyte formation, joint inflammation, and loss of normal joint function) that can culminate in illness" [1]. Chondropathy and osteogenesis occurring simultaneously are the main characteristic features of $\mathrm{OA}$ that reshape the knee joint contours. The conventional risk factors associated with $\mathrm{OA}$ are age, gender, body weight, vocation, 
sports, history of trauma, etc. In the global scenario, both hip and knee osteoarthritis are major causes of pain and disability and thus a compromised quality of life affecting the worldwide population. In OA, the articular cartilage undergoes changes such as softening, splitting, and fragmentation. The arthritic changes at the bony level include cystic cavities, microfractures, and osteophyte formation. The decrease in synovial fluid observed in $\mathrm{OA}$ is due to membrane hypertrophy, inflammation, and fibrotic degradation. These changes in turn affect the articular cartilage, surrounding capsule, ligaments, and also muscle atrophy. Thus, the symptoms of OA knee predominantly include pain, medial joint tenderness, reduction in range of motion, inflammation, knock knees, or bow legs [2-5]. Rehabilitation of patients with OA knee, hence, poses a challenge to health professionals.

With advances in research methods and technology, abundant evidence is available for different methods used during the treatment of $\mathrm{OA}$ knee ensuring that conventional methods are worthwhile in reducing the use of pain medications and coping with the multi-year gap between the diagnosis of OA knee, worsening of the condition due to various co-morbidities, and eventually joint replacement surgeries [6, 7]. Many studies recommend walking programs to rehabilitate patients with OA knee including aerobic walking, retro walking, and walking on a treadmill. Out of these, retro walking or backward walking is an exceptional proposal for the treatment of OA knee patients. Several benefits have been noted for retro walking such as different muscle activation, reduction in compression loads, changed direction of forces, and diverse joint mechanics. Retro walking as an adjunct to conventional therapy has proved significantly better in diminishing the disability and improving quality of life [7-12].

The other treatment maneuver that is also quite popular in the rehabilitation of OA knee is the balance training exercise. One of the complex processes of the human body that involves various neuromuscular events is balance. Balance is dictated by numerous sensory inputs from the vestibular, visual, and somatosensory systems. These inputs are centrally processed to generated coordinated responses ensuring that the center of mass always remains within the base of support. Balance control depends on accurate sensory input as well as the timely response of the appropriate muscles initiated by the proprioceptors. In OA knee, the joint reactivity resulting in inflammation and pain modifies the afferent impulses regarding joint movement and position. Thus, the proprioception is affected with resultant balance impairment. One of the consequences experienced due to this is the inappropriate muscle activation affecting the dynamic balance leading to functional instability. Pain also reduces the weight-bearing on the affected side causing the center of mass to shift away from the base of support further compromising the balance. Adequate balance is essential for maintaining postural stability during walking, stair climbing, and other activities of daily living. Takacs et al. in their study reported that dynamic balance training exercises benefitted OA knee patients by the significant reduction in pain, kinesiophobia, and improvement in their physical function. He-Sang et al. also found that patients with moderate and severe OA demonstrate increased balance deficits than those with mild OA [13-19].

Considering the extensive literature available on this subject, the aim of this study was to determine the effect of retro walking and balance training in the rehabilitation of patients with osteoarthritis of the knee. The objective of this study was to compare retro walking and balance training to evaluate which of these treatment protocols proved to be more efficient in managing pain and functional disability of patients with OA knee.

\section{Methods}

All procedures in this study were conducted in compliance with the institutional ethical approval from MGM College of Physiotherapy, Navi Mumbai (MGM/COP/ IRRC/123/2017). The patients recruited from the Physiotherapy Outpatient Department of the MGM College of Physiotherapy, Navi Mumbai, were explained about the study and a written informed consent was obtained from them. The participants were free to withdraw from the study at any time without any consequences. This study adheres to the CONSORT guidelines. This trial is retrospectively registered with the University Hospital Medical Information Network Centre (UMIN-CTR) UMIN000044483 on 2021/06/09.

\section{Study design}

This experiment was a single-blinded, three-arm randomized controlled clinical trial. The participants were randomly assigned to three groups-group A, control group receiving conventional therapy; group $\mathrm{B}$, receiving conventional therapy and retro walking training; and group $\mathrm{C}$, receiving conventional therapy and balance training. The participants were blinded and did not know which group they would be allotted to. Simple random sampling with the use of chits with group $\mathrm{A}$, group $\mathrm{B}$, and group $\mathrm{C}$ was used to assign the participants into the three groups by a physiotherapist when they were eligible and agreed to participate in the study. This protocol was conducted with an intention to treat.

\section{Sample size}

The sample size was calculated with a power of $80 \%$ and a level of significance of 0.05 (two-tailed) with a 
standard deviation based on the previously published study by Rathi et al. [20] yielding 60 patients who participated in the study with 20 patients assigned to each group.

\section{Study setting: physiotherapy outpatient department of the \{BLINDED FOR PEER REVIEW\} \\ Participants}

The patients with OA knee reporting to the Physiotherapy OPD were recruited in this study. The patients included in the study were within the age group of 45 to 60 years who visited the Physiotherapy OPD, reporting unilateral clinically diagnosed OA knee based on the ACR criteria [21]. This study excluded patients with severe pain (greater than 7 on the Numerical Pain Rating Scale), obesity (body mass index greater than $25 \mathrm{~kg} / \mathrm{m}^{2}$ ), history of lower limb trauma or surgery in the last 6 months, congenital deformities, other systemic arthritis, and also difficulty in walking for $10 \mathrm{~min}$ continuously. The study protocol is described in Fig. 1.

\section{Procedure}

Once the patients were recruited for the study, their basic demographic data was collected. After the participants were allocated to their respective groups, they performed warm-up exercises, followed by the intervention protocol designed for each group and then cool down exercises which included general body stretching and breathing exercises. The exercises were supervised by a trained physiotherapist 3 days per week for 6 weeks. Patients were instructed to note any adverse events such as increased pain, inflammation, and reduced range of motion and report immediately.

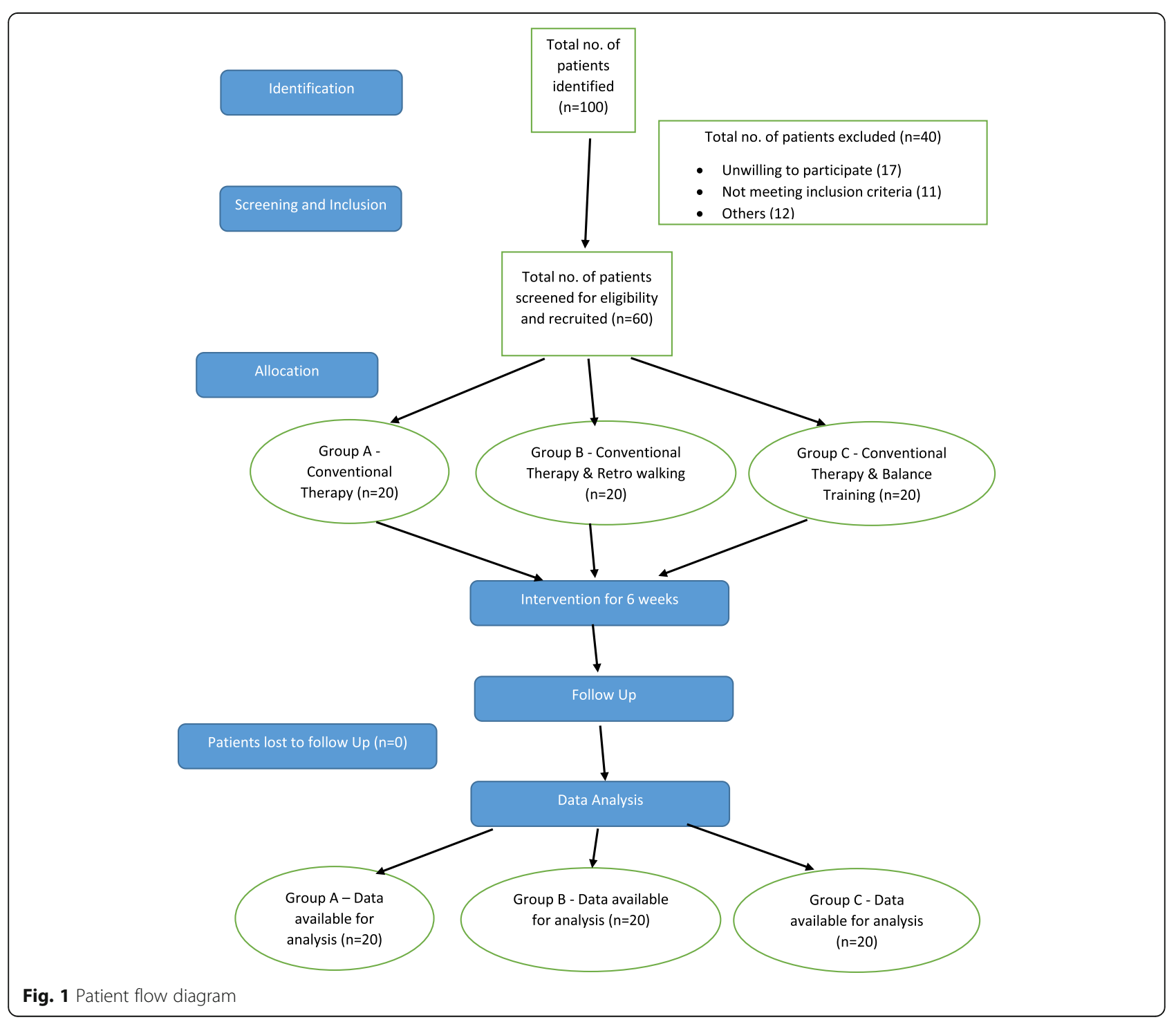




\section{Group A}

Group A received conventional therapy which included exercises such as static quadriceps, static hamstrings, strengthening of the hip abductors and extensors, straight leg raises, terminal knee extension, dynamic quadriceps exercises, and partial wall squats. Each exercise was performed for a single set of 10 repetitions twice a day for the first week and then progressed to 2 sets up to 3 weeks and finally to 3 sets up to 6 weeks.

\section{Group B}

Group B received conventional therapy and retro walking training. After the conventional exercises, the patients were instructed to walk backwards for $10 \mathrm{~min}$ on a flat surface for a distance of $30 \mathrm{~m}$ at a comfortable speed supervised by the physiotherapist. Patients were required to walk backwards without looking behind. Safety measures were taken to ensure patients' well-being during retro walking. The physiotherapist stood beside the patients, gave them moral support, and guided them through the walking path till the patients are confident to walk on their own. The patient is also encouraged to increase their speed throughout the 6 weeks of rehabilitation.

\section{Group C}

Group $\mathrm{C}$ received conventional therapy and balance training. Balance training followed the conventional exercises. The exercises consisted of standing in a wide base of support progressed to standing with a narrow base of support, perturbations, and reach-outs and tandem stance. Each of these exercises was first carried out with eyes open and then eyes closed. Static postures were maintained for $30 \mathrm{~s}, 1$ set initially then progressed to 3 sets by the 6 th week.

\section{Outcome measures}

Pain and functional disability of the patients were assessed by the physiotherapist using Intermittent and constant osteoarthritis pain measure (ICOAP) and Ibadan knee/hip osteoarthritis measure (IKHOAM). The patients were evaluated at the baseline and then at the end of the intervention at 6 weeks.

\section{Intermittent and constant osteoarthritis pain measure (ICOAP) [22, 23]}

ICOAP is a new tool designed to evaluate pain in hip/ knee osteoarthritis. In this study, the ICOAP knee version was used. It is an 11-item tool intended to respond to recent pain, i.e., pain in the last 7 days. It consists of two sections-constant pain and intermittent pain. The scores of the two together account for the total pain reported by the patient. The items are scored from " 0 to 4" on a Likert scale. The score obtained was then converted into a percentage value. The higher the percentage, the greater is the pain. This scale is a validated tool adapted and translated in many languages across the globe [24].

\section{Ibadan knee/hip osteoarthritis measure (IKHOAM) [25-27]}

IKHOAM was originally developed as a friendly tool for Nigerian patients. It is a 33-item instrument with 3 domains-activity limitation domain consisting of 25 items, participation restriction domain comprising 3 items (both are patient-rated), and the last physical performance domain with 5 tests rated by the clinician that includes walk test, stance test, stair climbing, squat test, and balance test. Each domain is scored separately, and the total thus obtained is deduced to percentages. Lower score indicates low physical function. Both validity and reliability of this scale are measured.

\section{Data analysis}

Data analysis was done using the SPSS software version 24. Demographic data, mean, and standard deviation for all the results were recorded. Descriptive statistics were used to analyze the groups. Normality was tested using the Shapiro-Wilk test resulting in freely distributed data. The Wilcoxon signed rank test was applied for intragroup comparison, and the Kruskal-Wallis test with Bonferroni correction post hoc analysis was applied for inter-group comparison. The level of significance was set at $p$ value $<0.05$ with $95 \%$ confidence interval.

\section{Results}

All the 60 OA knee patients recruited participated and completed the 6-week study protocol. None of the patients in the 3 groups reported any adverse events during the study duration.

\section{Discussion}

OA knee and its associated impairments continue to be surrounded by an ever-increasing enigma. Bearing in mind the social dynamics of our society, these complications result in functional affection especially for aging adults. This impacts the universal community disease dynamics. With the absence of a cure, the treatment of OA knee primarily focuses on pain relief, functional independence, and an enriched quality of life. To improve an individual's daily tasks, the Osteoarthritis Research Society International (OARSI) guidelines recommend regular walking programs for OA knee patients. It is proved that walking improves pain, functional capacity, and knee muscle activity [28, 29]. Also, several researchers have highlighted that OA knee alters joint proprioception and thus creates balance impairments in individuals. They conclude that balance exercises help restore the neuromuscular coordination and mend the 
Table 1 Baseline characteristics of participants

\begin{tabular}{llllll}
\hline Variables & All & Group A & Group B & Group C & p value \\
\hline Mean (SD) age, years & $56.38(4.96)$ & $57.55(5.37)$ & $55.30(4.70)$ & $56.30(4.79)$ & 6.14 \\
Gender (M/F) & $20 / 40$ & $6 / 14$ & $8 / 12$ & $24.92(2.10)$ & $24.79(2.02)$ \\
Mean (SD) BMI, kg/m² & $24.94(2.09)$ & $25.12(2.24)$ & $34.51(12.83)$ & $33.95(11.08)$ & 0.74 \\
Mean (SD) ICOAP & $32.13(11.46)$ & $27.95(9.62)$ & $64.03(7.68)$ & $63.53(10.48)$ \\
Mean (SD) IKHOAM & $64.44(8.62)$ & $65.78(7.67)$ & 0.17 \\
\hline
\end{tabular}

Inference: The baseline values for all the variables assessed across the three groups showed non-significant differences. It was noted that the study population consisted of $66.66 \%$ women

kinesthetic status [30, 31]. The evidence presented demonstrates the benefits of both retro walking and balance training for the rehabilitation of OA knee patients. The present study therefore was conducted to give an insight into the effects of retro walking and balance training as adjuncts to the conventional exercise protocol.

The baseline characteristics of the participants are illustrated in Table 1 . The variables across the groups showed statistical insignificance demonstrating the subject pool similarities. It was also noted that more than half of the study population were women. The results of the current study demonstrated that all the three protocols used for rehabilitation of OA knee benefitted the patients when the study parameters were compared pre- and post- 6 weeks observed in Table 2. This study established that retro walking along with conventional therapy more effectively improved the performance of OA knee patients with respect to pain and functional disability when compared to conventional therapy but was found to be equally effective in comparison with conventional therapy and balance training as illustrated in Tables 3 and 4. This study also exhibited that both conventional therapy and conventional therapy and balance training were not superior to each other.

The three programs implemented for OA knee rehabilitation were designed to achieve greater patient autonomy and independence. Conventional therapy is the standardized protocol used to treat OA knee patients on a regular basis. These exercises were specifically aimed at mobility, flexibility, and strength domains facilitating a reduction in the pain and functional disability. Thus, a statistically significant result was noted post- 6 weeks of the intervention.

Due to convincing research, the importance of balance and proprioception has been addressed in this study too. Target-specific training was introduced to conventional therapy for focused enhancement of balance. Ahmed [32], Tsauo et al. [33], and Moitra and Sharma [34] were among many others who recognized the positive effects of balance restoration in OA knee patients. Hee-Sang Kim et al. [19] in their study concluded that as the severity of the OA amplified, balance control was also found to be affected and recommended that rehabilitation of OA knee patients should incorporate balance training as a part of their regular protocol. These evidences support the findings of the current study and the importance of balance exercises. Although it was not found to be better than the other two protocols used, balance training can still be applied to rehabilitate patients with OA knee. The difference would have possibly emerged with statistical significance if the study population included elderly individuals. The evidence gathered was not strong enough to determine the superiority of one protocol over the other although both show improvements individually. This is probably due to the sample size evaluated in the study.

Walking is a primary function of humans and an activity that has been developed and perfected through decades. It is a commonly recommended, safe mode of exercise. Even with this, backward walking is an unusual task for the majority of individuals.

Table 2 Intragroup analysis for pain and functional disability using Wilcoxon signed ranks test

\begin{tabular}{|c|c|c|c|c|c|c|}
\hline \multirow[t]{2}{*}{ Variables } & \multicolumn{2}{|l|}{ Group A } & \multicolumn{2}{|l|}{ Group B } & \multicolumn{2}{|l|}{ Group C } \\
\hline & Mean (SD) & $p$ value & Mean (SD) & $p$ value & Mean (SD) & $p$ value \\
\hline ICOAP pre & $27.95(9.62)$ & $0.00^{*}$ & $34.51(12.83)$ & $0.00^{*}$ & 33.95 (11.08) & $0.00^{*}$ \\
\hline ICOAP post & $18.65(6.55)$ & & $12.46(4.58)$ & & $19.17(8.29)$ & \\
\hline IKHOAM pre & $65.78(7.67)$ & $0.00^{*}$ & $64.03(7.68)$ & $0.00^{*}$ & 63.53 (10.48) & $0.00^{*}$ \\
\hline IKHOAM post & $71.21(8.80)$ & & 77.57 (9.41) & & 73.15 (8.89) & \\
\hline
\end{tabular}

Inference: All the 3 treatment protocols showed statistically significant improvement for both pain and functional disability at the end of 6 weeks

* represents statistical significance 
Table 3 Intergroup analysis for pain and functional disability using the Kruskal-Wallis test

\begin{tabular}{llllll}
\hline Variables & \multicolumn{2}{l}{ Mean rank } & & $\begin{array}{l}\text { Chi- } \\
\text { square }\end{array}$ & $\begin{array}{l}\boldsymbol{p} \\
\text { value }\end{array}$ \\
\cline { 2 - 4 } & Group A & Group B & Group C & & \\
\hline ICOAP & 19.40 & 40.03 & 32.08 & 14.20 & $0.001^{*}$ \\
IKHOAM & 18.93 & 40.68 & 31.90 & 15.70 & $0.000^{*}$
\end{tabular}

Inference: Both pain and functional disability showed a statistically significant response to the rehabilitation protocol administered

* represents statistical significance

It involves reversal of leg movements, modifications in trajectories, and completely diverse biomechanics in comparison with forward walking. Researchers have found the many beneficial effects of backward walking such as enhanced knee extensor muscle activation and proprioception, muscle reflex initiation, change in motor control pattern, reduced gait speed and stride length, diminution in knee joint stresses, and improved hamstring and back muscle flexibility [35, 36]. The reversal of movement in retro walking results in greater demand placed on the ankle joint to assist in foot clearance, especially with the role reversal of the muscles to extensor activation pattern. Further, the external knee adduction moment during forward walking that leads to shear forces and compression of the medial compartment of the knee is counteracted during retro walking. Biomechanical analysis and EMG studies reveal that the hip and knee extensor muscles complement the ankle muscles resulting in foot contact point to ground reaction forces. This reduces the impact of the forces on the knee joint particularly the compressive forces on the patella. In addition to this, retro walking has also been proved to improve neuromuscular balance and coordination $[11,37,38]$. These mechanisms have greatly contributed to pain reduction and improvement in functional performance of the OA knee patients which is evident in our study.

\section{Conclusion}

Although, conventional therapy and balance training both prove to be assets, retro walking is established to

Table 4 Pairwise comparisons of the groups

\begin{tabular}{llll}
\hline Variables & Group A-group B & Group B-group C & Group A-group C \\
\hline ICOAP & $0.001^{*}$ & 0.450 & 0.065 \\
IKHOAM & $0.000^{*}$ & 0.336 & 0.056 \\
\hline
\end{tabular}

Inference: Post hoc analysis with Bonferroni correction shows that conventional therapy with retro walking (group B) showed a statistical significance compared to only conventional therapy (group A) implying the superiority of this protocol over the conventional therapy by itself. However, statistical significance was not observed on comparing conventional therapy with retro walking (group B) and conventional therapy with balance training (group C). Similar findings were demonstrated in comparison with conventional therapy (group A) with conventional therapy and balance training (group C)

* represents statistical significance be an excellent adjunct to conventional therapy for the rehabilitation of OA knee patients during the period of 6 weeks. Looking at the multifold virtues of retro walking, it is recommended to utilize this exercise as a part of our daily walking activity. The community and human society on the whole would immensely profit as an end result.

\section{Abbreviations \\ OA: Osteoarthritis; ACR criteria: American College of Rheumatology criteria; ICOAP: Intermittent and constant osteoarthritis pain measure; \\ IKHOAM: Ibadan knee/hip osteoarthritis measure; OARSI: Osteoarthritis Research Society International}

\section{Acknowledgements}

We would like to thank Dr. Adesola Odole for permitting us to use the Ibadan Knee/Hip Osteoarthritis Outcome Measure (IKHOAM) for our study. We would also like to express our gratitude to the principal, MGM College of Physiotherapy, for allowing this project and lastly all the wonderful patients who agreed to participate in our study.

\section{Authors' contributions}

Dr. Vijaya Krishnan (PT) has conceived the concept, formulating the protocol, performing the statistics, designing the analysis, and writing the paper. Dr. Krishna Pithadia (PT) contributed towards the concept, presented the idea, collected the data, performed the data analysis, and wrote the initial draft of the paper. Both the authors have read and approved the final manuscript.

\section{Funding}

This project has not received any funding.

Availability of data and materials

Yes, and will be disclosed on proper request

\section{Declarations}

\section{Ethics approval and consent to participate}

This study was approved by the Mahatma Gandhi Mission's College of Physiotherapy Institutional Research Review Committee (MGM/COP/IRRC/ 123/2017). Written informed consent was obtained from the subjects participating in the study.

Consent for publication

Not applicable.

\section{Competing interests}

The authors declare that they have no competing interests.

Received: 8 January 2021 Accepted: 20 June 2021

Published online: 20 October 2021

\section{References}

1. Kraus VB, Blanco FJ, Englund M. Karsdal, Lohmander LS. Call for standardized definitions of osteoarthritis and risk stratification for clinical trials and clinical use. Osteoarthr Cartil. 2015;23(8):1233-41. https://doi.org/10.1016/j.joca.2015. 03.036.

2. Khatri SM. Basics of orthopedic physiotherapy. New Delhi: JAYPEE Brothers Medical Publishers; 2013. chapter no. 6 Arthritis, 119-126

3. Arya RK, Jain V. Osteoarthritis of the knee joint: an overview. J Indian Acad Clin Med. 2013;14(2):154-62.

4. Symmons D, Mathers C, Fleger BP. Global burden of osteoarthritis in the year 2000. WHO Glob Burden Dis. 2003. Available from: http://www.who.int/ healthinfo/statistics/bod_osteoarthritis.pdf.

5. Cross M, Smith E, Hoy D, Nolte S, Ackerman I, Fransen M, et al. The global burden of hip and knee osteoarthritis: estimates from the global burden of disease 2010 study. Ann Rheum Dis. 2014;73(7):1323-30. https://doi.org/1 0.1136/annrheumdis-2013-204763.

6. Fransen M, McConnell S, Harmer AR, Van der Esch M, Simic M, Bennell KL. Exercise for osteoarthritis of the knee. In: The cochrane collaboration, editor. 
cochrane database of systematic reviews. Chichester: Wiley; 2015. Available from: http://doi.wiley.com/10.1002/14651858.CD004376.pub3.

7. Hochberg MC, Altman RD, April KT, Benkhalti M, Guyatt G, McGowan J, et al. American College of Rheumatology 2012 recommendations for the use of nonpharmacologic and pharmacologic therapies in osteoarthritis of the hand, hip, and knee. Arthritis Care Res. 2012;64(4):465-74. https://doi.org/1 $0.1002 /$ acr.21596.

8. Loew L, Brosseau L, Wells GA, Tugwell P, Kenny GP, Reid R, et al. Ottawa Panel evidence based clinical practice guidelines for aerobic walking programs in the management of osteoarthritis. Arch Phys Med Rehabil. 2012;93(7):1269-85. https://doi.org/10.1016/j.apmr.2012.01.024.

9. MCAlindon TE, Bannuru RR, Sullivan MC, Arden NK, Berenbaum F, BiermaZeinstra SM, et al. OARSI guidelines for the non-surgical management of knee osteoarthritis. Osteoarthr Cartil. 2014;22(3):363-88. https://doi.org/10.1 016/j.joca.2014.01.003.

10. Wadhwa DN, Hande DN. Effects of retro walking on osteoarthritis of knee in geriatric population. J Sports Phys Educ. 2016;3(2):37-43.

11. Balraj AM, Kutty RK, Kamraj B, Saji VT. Impact of retro walking on pain and disability parameters among chronic osteoarthritis knee patients. J Physiother Phys Rehabil. 2018;3(2):157-64.

12. Krishnan V, Goswami S. Effect of transcutaneous electrical nerve stimulation on knee joint proprioception - a cross sectional study in healthy adults. Int J Health Sci Res. 2018:8(8):171-5.

13. Bello A, Ababio E, Baffoe S, Seidu M, Adjei DN. Pain, range of motion and activity level as correlates of dynamic balance among elderly people with musculoskeletal disorder. Ghana Med J. 2014;48(4):214-8. https://doi.org/10.4314/gmj.v48i4.8.

14. Hurley M, Scott D, Rees J, Newham D. Sensorimotor changes and functional performance in patients with knee osteoarthritis. Ann Rheum Dis. 1997; 56(1):641-8. https://doi.org/10.1136/ard.56.11.641.

15. Rodriguez S, Rebolledo G, Munoz E, Palma P. The effect of progressive neuromuscular training on postural balance and functionality in elderly patients with knee osteoarthritis; a pilot study. J PhysTher Sci. 2017;29(7): 1229-35. https://doi.org/10.1589/jpts.29.1229.

16. Kyung K, Hae-Yong L, Sung-Joon L. Effects of increased standing balance on pain in patients with knee osteoarthritis. J Phys Ther Sci. 2016;28(1):87-9.

17. Knoop J, Steultjens M, Leeden M, Esch M, Thorstensson CA, Roorda LD, et al. Proprioception in knee osteoarthritis: a narrative review. Osteoarthr Cartil. 2011;19(4):381-8. https://doi.org/10.1016/j.joca.2011.01.003.

18. Takacs J, Krowchuk NM, Garland SJ, Carpenter MG, Hunt MA. Dynamic balance training improves physical function in individuals with knee osteoarthritis: a pilot randomized control trial. Arch Phys Med Rehabil. 2017; 98(8):1586-93. https://doi.org/10.1016/j.apmr.2017.01.029.

19. Hee-Sang K, Dong HY, Seung DY, Dong HK, Yong SJ, Jee-Sang Y, et al. Balance control and knee osteoarthritis severity. Ann Rehabil Med. 2011; 35(5):701-9.

20. Rathi M, Palekar T, Varghese A. Efficacy of backward walking on patients with osteoarthritis of knee on quadriceps strength, pain, and physical functions. Indian J Physio ther Occup Ther. 2014;8(4):192-6. https://doi.org/1 0.5958/0973-5674.2014.00036.7.

21. Damen J, Rijn RM, Emans PJ, Hilberdink WKHA, Wesseling J, Oei EHG, et al. Prevalence and development of hip and knee osteoarthritis according to American College of Rheumatology criteria in the CHECK cohort. Arthritis Res Ther. 2019;21(1):4-10. https://doi.org/10.1186/s13075-018-1785-7.

22. Hawker GA, Stewart L, French MR, Cibere J, Jordan JM, March L, et al. Understanding the pain experience in hip and knee osteoarthritis-an OARSI/OMERACT initiative. Osteoarthr Cartil. 2008;16(4):415-22. https://doi. org/10.1016/.joca.2007.12.017.

23. Goncalves RS, Meireles AC, Gil JN, Cavalheiro LM, Rosado JO, Cabri J. Responsiveness of intermittent and constant osteoarthritis pain (ICOAP) after physical therapy for knee osteoarthritis. Osteoarthr Cartil. 2012;20(10): 1116-9. https://doi.org/10.1016/j.joca.2012.06.013.

24. Mehta SP, Sankar A, Venkataramanan V, Lohmander LS, Katz JN, Hawker GA, et al. Cross-cultural validation of the ICOAP and physical function short forms of the HOOS and KOOS in a multi-country study of patients with hip and knee osteoarthritis. Osteoarthr Cartil. 2016;24(12):2077-81. https://doi. org/10.1016/j.joca.2016.07.014.

25. Odole AC, Odunaiya NA, Akinpelu AO. Ibadan knee/hip osteoarthritis outcome measure: process of development. Ann lb Postgrad Med. 2013;1 (2):71-8.

26. Akinpelu AO, Odole AC, Adegoke BOA, Adeyini AF. Development and initial validation of the Ibadan knee/hip osteoarthritis outcome measure. South Afr J Physiother. 2007;63(2):3-8.
27. Akinpelu AO, Akinwola MO, Odole AC, Gbiri CAO. The reliability of the English version of the Ibadan knee/hip osteoarthritis outcome measure (IKHOAM). Phys Occup Ther Geriatr. 2011;29(3):181-8. https://doi.org/10.31 09/02703181.2011.589566.

28. Zhang W, Moskowitz RW, Nuki G, Abramson S, Altman RD, Arden N, et al. OARSI recommendations for the management of hip and knee osteoarthritis, part II: OARSI evidence based, expert consensus guidelines. Osteoarthr Cartil. 2008;16(2):137-62. https://doi.org/10.1016/j. joca.2007.12.013.

29. White DK, Locke C, Felson DT, Gross KD, Niu J, Nevitt M, et al. Walking to meet physical activity guidelines in knee osteoarthritis: is 10,000 steps enough? Arch Phys Med Rehabil. 2013;94(4):711-7. https://doi.org/10.1016/ j.apmr.2012.11.038

30. Diracoglu D, Aydin R, Baskent A, Celik A. Effects of kinaesthesia and balance exercises in knee osteoarthritis. J Clin Rheumatol. 2005;11(6):303-10. https:// doi.org/10.1097/01.rhu.0000191213.37853.3d.

31. Ashtiani A, Akbari NJ, Mohammadi M, Nouraisarjou S. The effect of balance exercises on knee instability and pain intensity in patients with knee osteoarthritis: a randomized clinical trial. J Res Med Dent Sci. 2018;6(2):74-82.

32. Ahmed AF. Effect of sensorimotor training on balance in elderly patients with knee osteoarthritis. J Adv Res. 2011;2(4):305-11. https://doi.org/10.101 6/j.jare.2011.02.001.

33. Tsauo J, Cheng P, Yang R. The effects of sensorimotor training on knee proprioception and function for patients with knee osteoarthritis: a preliminary report. Clin Rehabil. 2008;22(5):448-57. https://doi.org/10.1177/ 0269215507084597

34. Moitra M, Sharma S. Effectiveness of proprioceptive training programme on joint position sense and balance in patients with knee osteoarthritis- a randomized control trial. J Disabil Stud. 2017;3(1):9-11.

35. Kachanathu SJ, Alabdulwahab SS, Negi N, Anand P, Hafeez AR. An analysis of physical performance between backward and forward walking training in young healthy individuals. Saudi J Sports Med. 2016;16(1):68-73. https://doi. org/10.4103/1319-6308.165112.

36. Joshi S, Vij JS, Singh SK. Retro walking: a new concept in physiotherapy and rehabilitation. Int J Sci Res. 2015;4(10):152-6.

37. Lee M, Kim J, Son J, Kim Y. Kinematic and kinetic analysis during forward and backward walking. Gait Posture. 2013;38(4):674-8. https://doi.org/10.101 6/j.gaitpost.2013.02.014.

38. Shankar P, Renukadevi M, Bhandiwa A, Pai H. Effectiveness of retro walking in chronic osteoarthritis of knee joint. Innov J Med Health Sci. 2013;3(1):19-22.

\section{Publisher's Note}

Springer Nature remains neutral with regard to jurisdictional claims in published maps and institutional affiliations.

\section{Submit your manuscript to a SpringerOpen ${ }^{\circ}$ journal and benefit from:}

- Convenient online submission

- Rigorous peer review

- Open access: articles freely available online

- High visibility within the field

- Retaining the copyright to your article

Submit your next manuscript at $\boldsymbol{\nabla}$ springeropen.com 